\title{
Owl Eye Appearance: Simultaneous Bilateral Hypertensive Putaminal Hematoma
}

\section{Sachin Baldawa*}

Department of Neurosurgery, Yashodhara Super speciality hospital, Solapur, Maharashtra, India

\begin{abstract}
Basal ganglia hemorrhage, one of the most devastating forms of cerebrovascular disease, is often as a result of poorly controlled long standing hypertension. Hypertension often leads to development of solitary intracerebral hematoma. The occurrence of multiple simultaneous intracerebral hemorrhages due to hypertension in same or different vascular territories is rare. We report the occurrence of simultaneous bilateral hypertensive putaminal hemorrhage appearing as mirror image (Owl eye appearance) in a 60 years old gentleman and discuss the probable pathophysiological mechanisms
\end{abstract}

Keywords: Intracerebral hemorrhage; Basal ganglia hemorrhage; Hypertensive hemorrhage; Mirror image

Abbreviation: ICH: Intracerebral Hemorrhage

\section{Introduction}

Intracerebral hemorrhage (ICH) accounts for 10 to $20 \%$ of all strokes. It is the leading cause of all strokes, second only to cerebral infarction [1]. Hypertension is the single most important risk factor for $\mathrm{ICH}$, often leading to solitary ICH $[2,3]$. Though hypertension can lead to recurrent $\mathrm{ICH}$, the occurrence of multiple simultaneous $\mathrm{ICH}$ is rare [4]. The occurrence of multiple simultaneous ICH has been observed in $2 \%$ of all hemorrhagic strokes [2,5]. Multiple simultaneous ICH due to hypertension has been reported in same or different vascular territories $[2,4]$. Simultaneous occurrence of hypertensive $\mathrm{ICH}$ in bilateral putaminal region appearing as mirror image (Owl eye appearance) has been rarely reported [5-7].

\section{Case report}

A 60 years old gentleman with a long history of untreated hypertension presented in the emergency department with sudden onset severe holocranial headache followed by acute onset deterioration in sensorium over a period of 2 hours. He was chronic smoker, smoking 1-2 packs/per day. His blood sugar was normal. At presentation his pulse was $66 / \mathrm{min}$, BP was $220 / 110 \mathrm{~mm} \mathrm{Hg}$. On neurological examination, the pupils were bilaterally equal and were reacting briskly to light. There was no eye opening to pain, he had left hemiplegia along with right hemiparesis. Bilateral plantar response was extensor. Fundus examination revealed grade 4 hypertensive changes. He was intubated and mechanically ventilated. He underwent computed tomography scan (CT) of the brain which revealed bilateral putaminal hematoma (Owl eye appearance). Left putaminal hematoma had extended into the left lateral ventricle (Figure 1). He was managed in Intensive stroke unit with anitedema measures and antihypertensives. Follow up CT scan done after 24 hours of stabilising the blood pressure did not reveal any increase in size of bilateral putaminal hematoma. Magnetic resonance angiogram of the brain done three days later did not reveal any vascular anomaly. He underwent tracheostomy and was gradually weaned off ventilator. After nearly four weeks at the time of discharge he remained in vegetative state.

\section{Discussion}

Hemorrhage in the lentiform nucleus or thalamus due to hypertension accounts for $35 \%-44 \%$ of cases of hypertensive ICH [5]. Hypertensive basal ganglia hemorrhage is one of the most devastating

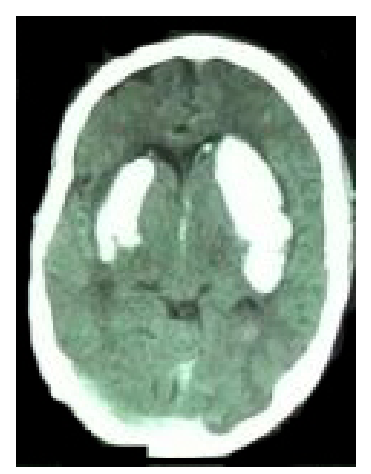

Figure 1: CT scan of the brain showing bilateral putaminal hemorrhage (Owl Eye appearance) with extension of the left putaminal hematoma in left lateral ventricle.

forms of cerebrovascular disease resulting in significant morbidity and mortality. It is often as a result of poorly controlled long standing hypertension. Hypertension is the most common etiological factor for the development of solitary ICH [4,5]. Hypertension often leads to the occurrence of recurrent ICH at same or different site [5-7]. Simultaneous occurrence of multiple ICH in different arterial territories is rare event. It has been reported following amyloid angiopathy, venous sinus thrombosis, coagulopathy, haemorrhagic transformation of cerebral infarcts and intracranial vascular anomalies or tumors [4,5]. However, the occurrence of multiple simultaneous ICH due to hypertension is uncommon $[2,5,6]$. Hypertensive bilateral putaminal hemorrhage causing mirror image (Owl eye appearance) is uncommon.

Long standing hypertension results in development of microaneurysms (Charcot-Bouchard aneurysms) of perforating arteries mainly the lenticulostriate and thalamoperforating arteries [2].

*Corresponding author: Sachin Baldawa, Department of Neurosurgery, Yashodhara Super Speciality Hospital, Solapur, Maharashtra, India, Tel: 91 9923406852; E-mail: sachin_baldawa@yahoo.co.in

Received: January 02, 2015; Accepted March 28, 2015; Published March 30, 2015

Citation: Baldawa S (2015) Owl Eye Appearance: Simultaneous Bilateral Hypertensive Putaminal Hematoma. J Neurol Disord 3: 225. doi: 10.4172/23296895.1000225

Copyright: (c) 2015 Baldawai S. This is an open-access article distributed under the terms of the Creative Commons Attribution License, which permits unrestricted use, distribution, and reproduction in any medium, provided the original author and source are credited. 
Citation: Baldawa S (2015) Owl Eye Appearance: Simultaneous Bilateral Hypertensive Putaminal Hematoma. J Neurol Disord 3: 225. doi: 10.4172/2329-6895.1000225

Page 2 of 2

Rupture of one of these microaneurysms on lenticulostriate arteries from proximal anterior cerebral artery and middle cerebral artery and thalamoperforating arteries from posterior cerebral artery often leads to solitary hemorrhage in putamen and thalamic region respectively [2]. Prolonged duration of hypertension leads to widespread degenerative changes of intraparenchymal arterioles resulting in development of simultaneous multiple ICH in same or different arterial territories $[2,4]$. Two possible mechanisms can be proposed for simultaneous development of bilateral hypertensive basal ganglia hematomas. One of them is simultaneous rupture of bilateral microaneurysms on lenticulostriate or thalamoperforating arteries [4]. However the most convincing theory is that the initial haemorrhage is unilateral which results in distortion of other parenchymal vessels coupled with reflex increase in blood pressure, in the setting of altered cerebral autoregulation, paves the way for second hemorrhage in short span on the contralateral side in patients with long standing uncontrolled hypertension $[2,4,6]$.

Majority of patients with simultaneous bilateral basal ganglia hematoma have poor outcome as compared to solitary ICH [4]. The poor outcome in these patients is due to destruction of crossing and non-crossing fibres, bilateral diaschisis phenomenon and development of severe disturbed consciousness, quadriparesis, and pseudobulbar palsy [4-7].

\section{Conclusion}

Simultaneous bilateral putaminal hemorrhage appearing as mirror image is rare, occurring due to uncontrolled long standing hypertension. The overall prognosis remains guarded due to poor neurological status and significant morbidity.

\section{References}

1. Feigin VL, Lawes CM, Bennett DA, Barker-Collo SL, Parag V (2009) Worldwide stroke incidence and early case fatality reported in 56 population-based studies: a systematic review. Lancet Neurol 8: 355-369.

2. Bevilacqua JA, Julio-R C, Dellarossa D (2005) Spontaneous bilatera hypertensive haemorrhage of the basal ganglia. Something more than CharcotBouchard aneurysms? Rev Neurol 41: 61-62.

3. O'Donnell MJ, Xavier D, Liu L, Zhang H, Chin SL, et al. (2010) Risk factors for ischaemic and intracerebral haemorrhagic stroke in 22 countries (the INTERSTROKE study): a case-control study. Lancet 376: 112-123.

4. Yen CP, Lin CL, Kwan AL, Lieu AS, Hwang SL, et al. (2005) Simultaneous multiple hypertensive intracerebral haemorrhages. Acta Neurochir 147: 393-399.

5. Kohshi K, Abe H, Tsuru E (2000) Simultaneous hypertensive intracerebral hematomas: two case reports. J Neurol Sci 181: 137-139.

6. Kabuto M, Kubota T, Kobayashi H, Nakagawa T, Arai Y, et al. (1995) Simultaneous bilateral hypertensive intracerebral hemorrhages--two case reports. Neurol Med Chir (Tokyo) 35: 584-586.

7. Asimi RP, Wani MA, Ahmad F (2007) Bilateral simultaneous hypertensive intracerebral hemorrhage in both putamen. Ann Indian Acad Neurol 10: 272-273. 